\title{
Measured Thermal Dissipation Field in Turbulent Rayleigh-Bénard Convection
}

\author{
Xiaozhou He, ${ }^{1}$ Penger Tong, ${ }^{1}$ and Ke-Qing $\mathrm{Xia}^{2}$ \\ ${ }^{1}$ Department of Physics, Hong Kong University of Science and Technology, Clear Water Bay, Kowloon, Hong Kong \\ ${ }^{2}$ Department of Physics, the Chinese University of Hong Kong, Shatin, Hong Kong \\ (Received 7 February 2006; revised manuscript received 31 December 2006; published 2 April 2007)
}

\begin{abstract}
The time-averaged local thermal dissipation rate $\epsilon_{N}(\mathbf{r})$ in turbulent convection is obtained from direct measurements of the temperature gradient vector in a cylindrical cell filled with water. It is found that $\epsilon_{N}(\mathbf{r})$ contains two contributions. One is generated by thermal plumes, present mainly in the plumedominated bulk region, and decreases with increasing Rayleigh number Ra. The other contribution comes from the mean temperature gradient, being concentrated in the thermal boundary layers, and increases with $\mathrm{Ra}$. The experiment thus provides a new physical picture about the thermal dissipation field in turbulent convection.
\end{abstract}

DOI: $10.1103 /$ PhysRevLett.98.144501

PACS numbers: 47.27.T,$- 44.25 .+\mathrm{f}$

Turbulent Rayleigh-Bénard convection in a fluid layer confined between two horizontal plates of separation $H$ occurs when the Rayleigh number Ra becomes sufficiently large. Here $\mathrm{Ra}$ is defined as $\mathrm{Ra}=\alpha g \Delta T H^{3} /(\nu \kappa)$, where $g$ is the gravitational acceleration, $\Delta T$ is the temperature difference between the lower heated and upper cooled plates, and $\alpha, \nu$, and $\kappa$ are, respectively, the thermal expansion coefficient, the kinematic viscosity, and the thermal diffusivity of the convecting fluid. An important issue that has been under intensive experimental and theoretical scrutiny in recent years is to understand how heat is transported vertically through the convection cell $[1,2]$. A large number of heat transport measurements have been carried out in various convecting fluids with wide parameter range and great precision [3]. These measurements shed new light on the mechanism of heat transport and have stimulated considerable theoretical efforts, aimed at explaining the functional form of the measured Nusselt number $\mathrm{Nu}(\mathrm{Ra}, \mathrm{Pr})$ (normalized heat flux) as a function of two experimental control parameters: Ra and the Prandtl number $\operatorname{Pr}=\nu / \kappa$.

An quantity that is closely connected to $\mathrm{Nu}(\mathrm{Ra}, \mathrm{Pr})$ is the thermal dissipation field $\epsilon_{T}(\mathbf{r}, t)=\kappa|\nabla T(\mathbf{r}, t)|^{2}$, where $\nabla T(\mathbf{r}, t)$ is the temperature gradient field. The determination of $\epsilon_{T}(\mathbf{r}, t)$ involves simultaneous measurement of three components of $\nabla T(\mathbf{r}, t)$. Experimental studies of scalar dissipation fields have been carried out in turbulent flows [4], in which temperature (or concentration of a contaminant) is a passive scalar. In this case, $\epsilon_{T}(\mathbf{r}, t)$ measures a mixing rate, at which fluctuations of $T$ (or $T^{2}$ ) are destroyed. For thermal convection, however, temperature is an active scalar which drives the convective flow. In this case, $\epsilon_{T}(\mathbf{r}, t)$ is directly linked to the local dynamics of the flow and one finds [2] $\left\langle\epsilon_{N}(\mathbf{r}, t)\right\rangle_{V, t}=\mathrm{Nu}(\mathrm{Ra}, \mathrm{Pr})$, where $\epsilon_{N}(\mathbf{r}, t) \equiv \epsilon_{T}(\mathbf{r}, t) /\left[\kappa(\Delta T / H)^{2}\right]$ is the normalized thermal dissipation field, and $\langle\ldots\rangle_{V, t}$ represents averages over the fluid volume $V$ and time $t$.

The theory by Grossmann and Lohse (GL) [2,5] explains the scaling behavior of $\mathrm{Nu}(\mathrm{Ra}, \mathrm{Pr})$ by a decomposition of
$\epsilon_{N}(\mathbf{r})=\left\langle\epsilon_{N}(\mathbf{r}, t)\right\rangle_{t}$ into two parts. In one scenario [2], $\epsilon_{N}(\mathbf{r})$ is decomposed into the boundary-layer and bulk contributions, which have different scaling behavior with varying $\mathrm{Ra}$ and $\mathrm{Pr}$. More recently, a second scenario was proposed [5] with $\epsilon_{N}(\mathbf{r})$ being decomposed into two different contributions: thermal plumes (including the boundary layers) and turbulent background. While the two scenarios involve different physical pictures about the local dynamics of turbulent convection, the calculated $\mathrm{Nu}(\mathrm{Ra}, \mathrm{Pr})$ using the two different models turns out to be of the same scaling form. This suggests that while the GL theory is capable of providing a correct functional form of $\mathrm{Nu}(\mathrm{Ra}, \mathrm{Pr})$ for a large number of transport measurements [3], the microscopic mechanism of heat transport and its connection to the local dynamics of turbulent convection still remain illusive.

In this Letter, we report direct measurements of $\epsilon_{N}(\mathbf{r}, t)$ over varying Rayleigh numbers and spatial positions $\mathbf{r}$ across the convection cell. Four identical thermistors are used to make a small local temperature gradient probe. One of the thermistors is placed at the origin, labeled as $T_{0}$, and the other three are arranged along the $x, y$, and $z$ axes, respectively. By simultaneously measuring the four temperature signals, we obtain the three temperature gradient components $\delta T_{i} / \delta \ell$, where $\delta T_{i}=T_{i}-T_{0}(i=x, y, z)$ is the temperature difference between a pair of the thermistors with separation $\delta \ell$. Two temperature gradient probes are used in the experiment. One consists of four preassembled thermistors with $0.17 \mathrm{~mm}$ in diameter and $\delta \ell=$ $0.8 \pm 0.1 \mathrm{~mm}$. This probe is used to measure the spatial distribution of $\epsilon_{N}(\mathbf{r})$ at fixed values of Ra and its spatial resolution $\delta \ell$ is comparable to the thermal boundary-layer thickness $\delta\left(\simeq 0.8 \mathrm{~mm}\right.$ at $\left.\mathrm{Ra}=3.6 \times 10^{9}\right)$, which is the smallest length scale in turbulent convection. The second probe is made of four smaller thermistor beads of $0.11 \mathrm{~mm}$ in diameter and is assembled in our own lab. It has a higher spatial resolution with $\delta \ell=0.25 \pm 0.1 \mathrm{~mm}$. This probe is used to measure the Ra dependence of $\epsilon_{N}(\mathbf{r})$. All the thermistors are calibrated individually with an accuracy 
of $\sim 5 \mathrm{mK}$ for $\delta T_{i}$. Each thermistor is connected to an ac bridge as a resistance arm and four identical lock-in amplifiers are used, each operating at a slightly different reference frequency $f_{0} \simeq 1 \pm 0.2 \mathrm{kHz}$ to avoid cross-talks between the four signals. The sampling rate is set at $40 \mathrm{~Hz}$. Typically, we take $2-7 \mathrm{~h}$ long time series $\left(\sim 5 \times 10^{5}\right.$ data points) at each location, ensuring that the statistical average is adequate. The experiment is conducted in an upright cylindrical cell filled with water. The inner diameter of the cell is $D=19 \mathrm{~cm}$ and height is $H=20 \mathrm{~cm}$. Other details about the cell can be found in [6]. The mean temperature of the bulk fluid is kept at $\sim 30^{\circ} \mathrm{C}$ and thus $\operatorname{Pr} \simeq 5.4$.

We first discuss the measurements of $\epsilon_{N}(\mathbf{r})$ along the cell diameter at the midheight of the cell ( $x$ axis) and along the central vertical axis of the cell ( $z$ axis), both are in the rotation plane of the large-scale circulation. From the definition of $\epsilon_{N}(\mathbf{r})$, one finds that $\epsilon_{N}(\mathbf{r})$ contains three contributions, $\epsilon_{N}^{i}(\mathbf{r})(i=x, y, z)$, resulting from the three components of the temperature gradient. Each contribution can be further decomposed into two terms: $\epsilon_{N}^{i}(\mathbf{r})=$ $\boldsymbol{\epsilon}_{m}^{i}(\mathbf{r})+\boldsymbol{\epsilon}_{f}^{i}(\mathbf{r})$, with $\boldsymbol{\epsilon}_{m}^{i}(\mathbf{r})$ resulting from the mean temperature gradient and $\epsilon_{f}^{i}(\mathbf{r})$ coming from the fluctuations. As a result, the total dissipation can be written as $\epsilon_{N}(\mathbf{r})=$ $\epsilon_{m}(\mathbf{r})+\epsilon_{f}(\mathbf{r})$. The inset of Fig. 1(a) shows the measured

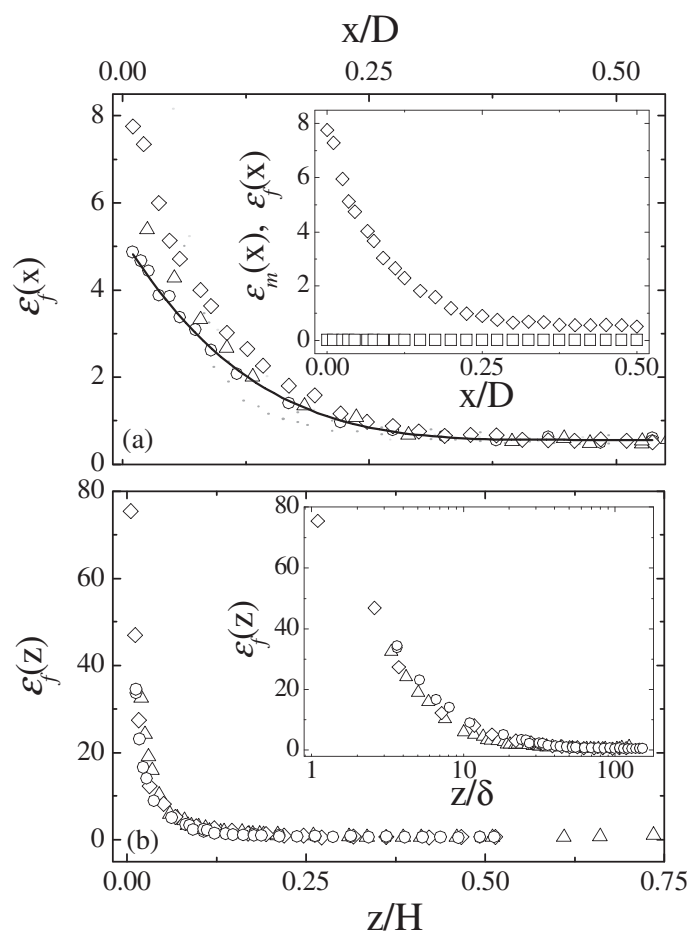

FIG. 1. (a) Measured horizontal profile $\epsilon_{f}(x)$ as a function of $x / D$. The solid line shows the fitted function, $\epsilon_{f}(x)=$ $a+b(x / D-0.5)^{4}$ with $a=0.56$ and $b=74.4$. The inset compares $\epsilon_{f}(x)$ (diamonds) with $\epsilon_{m}(x)$ (squares) at $\mathrm{Ra}=2.7 \times$ $10^{9}$. (b) Vertical profile $\epsilon_{f}(z)$ as a function of $z / H$. The inset shows the same data as a function of $z / \delta$. The measurements in (a) and (b) are made at $\mathrm{Ra}=9.2 \times 10^{8}$ (triangles), $2.7 \times 10^{9}$ (diamonds), and $6.6 \times 10^{9}$ (circles). $\epsilon_{m}(x)$ (squares) and $\epsilon_{f}(x)$ (diamonds) as a function of the normalized horizontal position $x / D(x / D=0.5$ at the cell center). It is seen that $\epsilon_{m}(x)$ is negligibly small when compared with $\epsilon_{f}(x)$. The conclusion that $\epsilon_{N}(\mathbf{r}) \simeq \epsilon_{f}(\mathbf{r})$ is found to be true for all the measurements in the bulk region outside the thermal boundary layer. Inside the thermal boundary layer, we find $\epsilon_{N}(\mathbf{r}) \simeq \epsilon_{m}(\mathbf{r})\left[\epsilon_{f}(\mathbf{r})\right.$ is less than $1.5 \%$ of $\left.\epsilon_{m}(\mathbf{r})\right]$.

Figure 1(a) shows the horizontal profiles of $\epsilon_{f}(x)$ for three different values of Ra. The measured $\epsilon_{f}(x)$ is symmetric about the central axis with a minimum value at the cell center and increases sharply in the sidewall region, in which both the local velocity and local convective heat flux reach maximum [7,8]. The value of $\epsilon_{f}(x)$ near the sidewall is $\sim 10$ times larger than that at the cell center. The amplitude of the horizontal profile $\epsilon_{f}(x)$ decreases slightly with increasing $\mathrm{Ra}$, but its general shape remains unchanged in the $\mathrm{Ra}$ range studied.

Figure 1(b) shows $\epsilon_{f}(z)$ as a function of the normalized vertical position $z / H$ for three different values of Ra. Similar to the horizontal scan, the measured $\epsilon_{f}(z)$ has a minimal value at the cell center $(z / H=0.5)$ and reaches maximum near the lower conducting plate. When compared with Fig. 1(a), we find that $\boldsymbol{\epsilon}_{f}(z)$ increases more rapidly near the conducting plate. As shown in the inset, the rise of $\epsilon_{f}(z)$ takes place mainly in the $1 \leqq z / \delta \leqq 10$ region, where $\delta=425 \mathrm{Ra}^{-0.285} \mathrm{~mm}$ [9]. The maximal value of $\epsilon_{f}(z)$ at $z / \delta \simeq 1$ is $\sim 140$ times larger than the minimal value at the cell center. Another important feature shown in the inset is that the rise of $\epsilon_{f}(z)$ near the conducting plate scales with $\delta$.

Figure 2 shows the measured horizontal profiles $\epsilon_{f}^{i}(x)$ as a function of $x / D$. In the sidewall region, $\epsilon_{f}^{z}(x)$ is approximately twice larger than $\epsilon_{f}^{x}(x)$ and $\epsilon_{f}^{y}(x)$. By carefully examining the time series data of the local temperature and temperature gradient, we find that the large value of $\boldsymbol{\epsilon}_{f}^{z}(x)$ comes from fluctuations of the temperature gradient produced by the rising (or falling) thermal plumes. Similarly, the vertical scan (not shown) reveals that the dominant contribution to $\epsilon_{f}(z)$ is from $\epsilon_{f}^{z}(z)$, which is

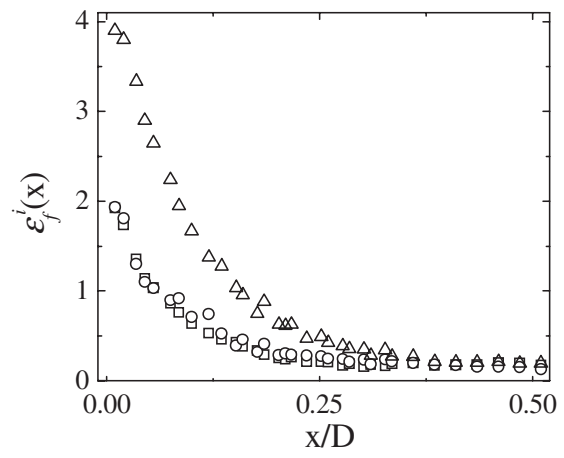

FIG. 2. Horizontal profiles $\epsilon_{f}^{x}(x)$ (squares), $\epsilon_{f}^{y}(x)$ (circles), and $\boldsymbol{\epsilon}_{f}^{z}(x)$ (triangles) as a function of $x / D$ at $\mathrm{Ra}=2.7 \times 10^{9}$. 
approximately twice larger than $\epsilon_{f}^{x}(z)$ and $\epsilon_{f}^{y}(z)$ near the boundary layer.

We now discuss the Ra dependence of $\epsilon_{N}(\mathbf{r})$ at several representative locations in the convection cell. Figure 3(a) shows $\epsilon_{f}(\mathbf{r})$ as a function of Ra at the cell center (circles), near the sidewall (triangles), and near the lower conducting plate (diamonds). Because of the large differences in amplitude, the values of the circles and triangles are multiplied by 200 and 25, respectively, in order to display them in the same graph. The measured $\epsilon_{f}(\mathbf{r})$ at the cell center and near the sidewall is well described by a power law $\boldsymbol{\epsilon}_{f}(\mathbf{r})=\alpha \mathrm{Ra}^{-\beta}$ (solid lines) with $\beta=0.33 \pm 0.03$ for both sets of the data. The power-law amplitude $\alpha$ for the circles is $1.9 \times 10^{3}$ and that for the triangles is $1.05 \times 10^{4}$. The measured $\epsilon_{N}(\mathbf{r})$ near the lower conducting plate first increases with $\mathrm{Ra}$ for small values of $\mathrm{Ra}$ and then decreases at larger values of $\mathrm{Ra}$. This is because the measuring position at small values of Ra is located inside the thermal boundary layer. As the value of Ra increases, the boundarylayer thickness decreases and the measuring position moves outside the boundary layer. The large-Ra portion of the data can be described by the same power law with $\alpha=2.05 \times 10^{5}$ and $\beta=0.33$ (solid line). To obtain the Ra dependence of $\epsilon_{m}(\mathbf{r})$ inside the boundary layer, we place the temperature gradient probe even closer to the lower conduction plate $(\sim 0.2 \mathrm{~mm}$ above the bottom plate) and the result is shown in Fig. 3(b). The solid line is a power-law fit, $\epsilon_{m}=1.1 \times 10^{-2} \mathrm{Ra}^{\gamma}$, with $\gamma=0.63 \pm 0.05$.

Because of finite heat capacity of the conducting plates and finite spatial resolution of the temperature gradient

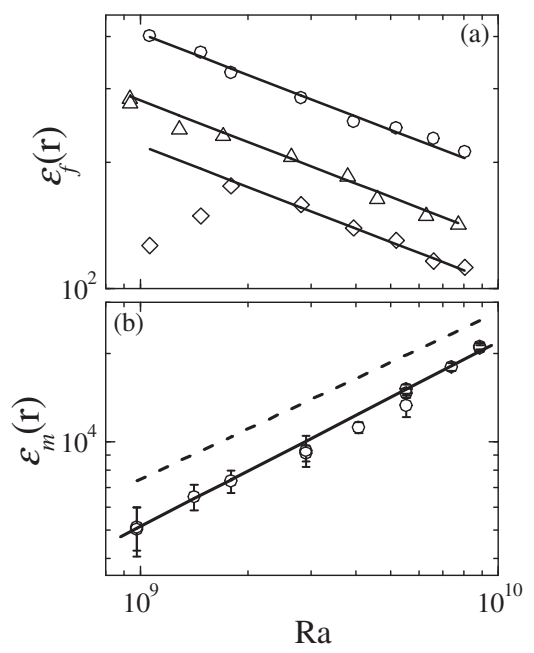

FIG. 3. (a) $\epsilon_{f}(\mathbf{r})$ as a function of Ra at the cell center (circles), near the sidewall (triangles), and near the lower conducting plate at distance $\sim 1 \mathrm{~mm}$ above the bottom plate (diamonds). The values of the circles and triangles have been multiplied by 200 and 25 , respectively. The solid lines show the power-law fits. (b) Ra dependence of $\epsilon_{m}(\mathbf{r})$ inside the thermal boundary layer ( $\sim 0.2 \mathrm{~mm}$ above the bottom plate). The solid line is a powerlaw fit, $\epsilon_{m}=1.1 \times 10^{-2} \mathrm{Ra}^{0.63}$. The dashed line shows $(H / 2 \delta)^{2} \simeq 5.54 \times 10^{-2} \mathrm{Ra}^{0.57}$ (see text). probe, the obtained value of $\gamma$ may have relatively larger uncertainties. However, the following physical picture about the thermal dissipation field in turbulent convection, which is derived from the above measurements, will not be affected by these experimental uncertainties. First, the thermal dissipation field can be divided into two regions. (i) In the bulk region outside the boundary layers, the dominant contribution to $\epsilon_{N}(\mathbf{r})$ comes from fluctuations of the local temperature gradient. The dissipation field $\epsilon_{f}(\mathbf{r})$ is generated by the detached thermal plumes and occupies mainly in the plume-dominated bulk region near the sidewall and the boundary layers $(z / \delta>1)$. (ii) Inside the thermal boundary layers $(z / \delta \lesssim 1)$, the dominant contribution to $\epsilon_{N}(\mathbf{r})$ comes from the mean temperature gradient in the direction perpendicular to the conducting surfaces.

While the spatial decomposition $\epsilon_{N}(\mathbf{r})=\epsilon_{f}(\mathbf{r})+\epsilon_{m}(\mathbf{r})$ is consistent with the first scenario of the GL theory [2], the measured Ra dependence of $\epsilon_{f}(\mathbf{r})$ and $\epsilon_{m}(\mathbf{r})$ does not agree with this scenario. It is found that $\epsilon_{f}(\mathbf{r})$ throughout the bulk region scales as $\mathrm{Ra}^{-0.33}$, whereas $\boldsymbol{\epsilon}_{m}(\mathbf{r})$ inside the boundary layers scales with Ra differently $\left(\epsilon_{m}(\mathbf{r}) \sim \mathrm{Ra}^{0.63}\right)$. This $\mathrm{Ra}$ dependence has two important implications. First, the volume average ratio $\left\langle\epsilon_{f}(\mathbf{r})\right\rangle_{V} /\left\langle\epsilon_{m}(\mathbf{r})\right\rangle_{V}$ will decrease with increasing $\mathrm{Ra}$, a trend which agrees with the recent numerical results [10] but is opposite to that given by the first scenario of the GL theory [2,5]. Second, the measurements clearly reveal two competing effects of turbulence. On one hand, temperature fluctuations are destroyed in the bulk region and their contributions to $\epsilon_{N}(\mathbf{r})$ decrease with increasing Ra. On the other hand, the temperature gradient (and hence the thermal dissipation) is enhanced near the conducting plates, because of the thinning of the boundary layers with increasing $\mathrm{Ra}$. These two competing effects thus suggest that thermal plumes and the boundary layers are two different dynamic structures and cannot be treated equally, as suggested by the second scenario of the GL theory $[2,5]$.

The dashed line in Fig. 3(b) shows the measured $(H / 2 \delta)^{2} \simeq 5.54 \times 10^{-2} \mathrm{Ra}^{0.57}$ [9], which has a larger amplitude but smaller exponent when compared with the measured $\epsilon_{m}(\mathbf{r})$. The two curves intersect at $\mathrm{Ra}_{c} \simeq 1.68 \times$ $10^{11}$, above which one expects that $\epsilon_{N}(\mathbf{r})$ will be dominated by the boundary-layer contributions. Indeed, using the simple boundary-layer scaling [2,5], one finds that $\boldsymbol{\epsilon}_{m}(\mathbf{r}) \simeq(H / 2 \delta)^{2}$ [which is an upper bound for $\epsilon_{m}(\mathbf{r})$ without taking fluctuations into account] and thus $\left\langle\epsilon_{m}(\mathbf{r})\right\rangle_{V} \simeq$ $(H / 2 \delta)^{2}(2 \delta / H) \simeq \mathrm{Nu}$ (i.e., the boundary layers account for all the contributions to $\mathrm{Nu}$ ). In fact, the recent heat transport measurements [3] have shown that $\mathrm{Nu} \sim \mathrm{Ra}^{1 / 3}$ for $\mathrm{Ra} \geq \mathrm{Ra}_{c}$, which is a classical scaling based on a simple boundary-layer argument [1,2]. The Ra dependence of $\epsilon_{f}(\mathbf{r})$ and $\epsilon_{m}(\mathbf{r})$ thus provides a microscopic explanation to this boundary-layer dominance transition at $\mathrm{Ra} \gtrsim \mathrm{Ra}_{c}$.

The above boundary-layer scaling arguments, however, neglect fluctuations of the boundary layer due to the de- 


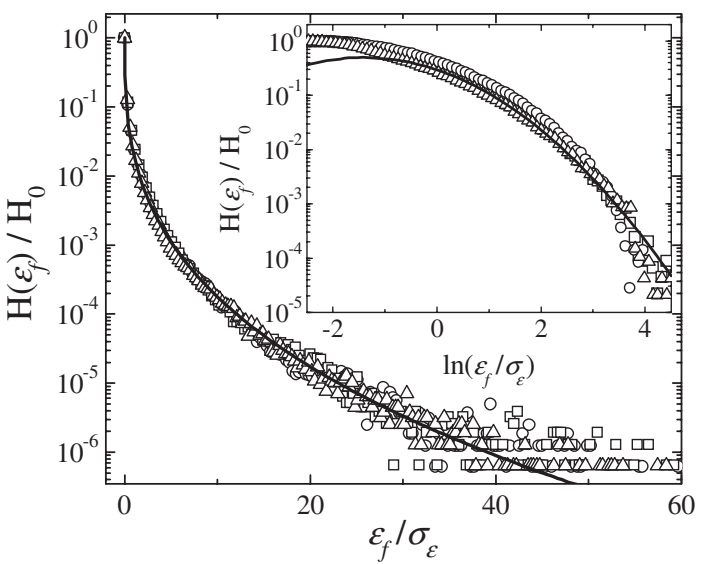

FIG. 4. Histograms $H\left(\epsilon_{f}\right) / H_{0}$ as a function of $\epsilon_{f} / \sigma_{\epsilon}$ at the cell center. The values of Ra are $9.6 \times 10^{8}$ (squares), $2.8 \times 10^{9}$ (triangles), and $8.2 \times 10^{9}$ (circles). The solid line shows the fitted function, $H\left(\epsilon_{f}\right) / H_{0}=\exp \left[-c\left(\epsilon_{f} / \sigma_{\epsilon}\right)^{d}\right]$, with $c=3.9$ and $d=0.35$. The inset shows the same data as a function of $\ln \left(\epsilon_{f} / \sigma_{\epsilon}\right)$. The solid line is a fit to the triangles with the lognormal distribution, $H\left(\epsilon_{f}\right) / H_{0}=\ell \exp \left\{-\left[\ln \left(\epsilon_{f} / \sigma_{\epsilon}\right)-\right.\right.$ $\left.m]^{2} / 2 n^{2}\right\}$, where $\ell=0.5, m=-1.4$ (mean) and $n=1.37$ (standard deviation).

tachment of thermal plumes, which can cause a (momentary) increase of the boundary-layer thickness (mostly occurring in the corner region of the convection cell $[7,9])$, resulting in a slight decrease of the thermal dissipation inside the boundary layers. The detached thermal plumes are then transported to and dissipated in the bulk region by the large-scale flow. As an estimate, we calculate the spatially averaged value $\left\langle\epsilon_{f}(\mathbf{r})\right\rangle_{V}$. By assuming $\epsilon_{f}(\mathbf{r}) d \mathbf{r} \simeq\left[\epsilon_{N}(x) \epsilon_{N}(z) / \epsilon_{N}(0)\right] 2 \pi x d x d z$ is axially symmetric, we find $\left\langle\epsilon_{f}(\mathbf{r})\right\rangle_{V} \approx 8.1$ at $\mathrm{Ra}=2.7 \times 10^{9}$. This accounts for $\sim 9.7 \%$ of the measured $\mathrm{Nu}$ at this $\mathrm{Ra}$ [9]. Because $\epsilon_{f}(\mathbf{r})$ decreases with increasing $\mathrm{Ra}$, the onset of the $\mathrm{Nu} \sim \mathrm{Ra}^{1 / 3}$ scaling at $\mathrm{Ra} \gtrsim \mathrm{Ra}_{c}$ may be viewed as a manifestation of diminishing contribution of $\epsilon_{f}(\mathbf{r})$ to the total dissipation rate.

In addition to the time-averaged properties, we also study the statistical properties of dissipation fluctuations $\epsilon_{f}(\mathbf{r}, t)$. Figure 4 shows the histograms $H\left(\epsilon_{f}\right)$ at the cell center. The histograms obtained at different Ra can all be brought into coincidence, once $H\left(\epsilon_{f}\right)$ is normalized by its maximum value $H_{0}$ and $\epsilon_{f}$ is scaled by its rms value $\sigma_{\epsilon}$. Plots of $H\left(\epsilon_{f}\right) / H_{0}$ vs $\epsilon_{f} / \sigma_{\epsilon}$ remain unchanged in the Ra range studied and only $\sigma_{\epsilon}$ changes with Ra. The histograms have a universal form, which can be described by a stretched exponential function over an amplitude range of almost five decades (solid curve). The inset shows that $H\left(\epsilon_{f}\right) / H_{0}$ can also be described by a log-normal distribu- tion for fluctuations of $\ln \left(\epsilon_{f} / \sigma_{\epsilon}\right)$ larger than their mean value $m \simeq-1.4$ (solid curve). The measured $H\left(\epsilon_{f}\right)$ near the sidewall (not shown) is of the same form. Log-normal distributions have been used to describe the intermittent nature of viscous dissipation fluctuations [11]. In fact, the cascade picture for the viscous dissipation field with rare localized regions of strong and/or weak energy dissipations may also apply to the thermal dissipation field in turbulent convection. Such localized regions in thermal convection can be naturally identified as thermal plumes, as evidenced by our finding that $\epsilon_{f}(\mathbf{r})$ is dominated by contributions from the plumes. It was also shown recently [12] that both the size and the "heat content" of thermal plumes exhibit log-normal distributions.

We thank D. Lohse and E. Ching for useful discussions. This work was supported by the Hong Kong RGC under Grants No. HKUST-CA05/06.SC01 (P.T.) and No. CUHK403003, 403705 (K. Q. X.).

[1] E. Siggia, Annu. Rev. Fluid Mech. 26, 137 (1994); L. P. Kadanoff, Phys. Today 54, No. 8, 34 (2001).

[2] S. Grossmann and D. Lohse, J. Fluid Mech. 407, 27 (2000); Phys. Rev. Lett. 86, 3316 (2001); Phys. Rev. E 66, 016305 (2002).

[3] B. Castaing et al., J. Fluid Mech. 204, 1 (1989); J. A. Glazier et al., Nature (London) 398, 307 (1999); X. Chavanne et al., Phys. Fluids 13, 1300 (2001); J. J. Niemela et al., Nature (London) 404, 837 (2000); J. Fluid Mech. 557, 411 (2006); G. Ahlers et al., Phys. Rev. Lett. 86, 3320 (2001); J. Fluid Mech. 536, 145 (2005); X.-Q. Xia et al., Phys. Rev. Lett. 88, 064501 (2002); C. Sun et al., J. Fluid Mech. 542, 165 (2005).

[4] K. R. Sreenivasan et al., Phys. Fluids 20, 1238 (1977); R. R. Prasad et al., Phys. Rev. Lett. 61, 74 (1988); D. Kushnir et al., Phys. Rev. Lett. 97, 124502 (2006).

[5] S. Grossmann and D. Lohse, Phys. Fluids 16, 4462 (2004).

[6] Y.-B. Du and P. Tong, J. Fluid Mech. 407, 57 (2000); X.-L. Qiu et al., Phys. Fluids 16, 412 (2004).

[7] X.-L. Qiu and P. Tong, Phys. Rev. E 64, 036304 (2001); C. Sun et al., Phys. Rev. E 72, 026302 (2005).

[8] X.-D. Shang et al., Phys. Rev. Lett. 90, 074501 (2003); Phys. Rev. E 70, 026308 (2004).

[9] S.-L. Lui and K.-Q. Xia, Phys. Rev. E 57, 5494 (1998).

[10] R. Verzicco and R. Camussi, J. Fluid Mech. 477, 19 (2003); R. Verzicco, Eur. Phys. J. B 35, 133 (2003).

[11] A. S. Gurvich and A. M. Yaglom, Phys. Fluids 10, S59 (1967); U. Frisch, Turbulence: The Legacy of A.N. Kolmogorov (Cambridge University Press, Cambridge, U.K., 1995).

[12] S.-Q. Zhou and K.-Q. Xia, Phys. Rev. Lett. 89, 184502 (2002); Q. Zhou et al., Phys. Rev. Lett. 98, 074501 (2007). 